\title{
Interchannel Crosstalk Reduction in an Analog Fiber Link Using Dispersion Management
}

\author{
Brian S. Marks, Member, IEEE, Curtis R. Menyuk, Fellow, IEEE, Anthony L. Campillo, Member, IEEE, and \\ Frank Bucholtz, Member, IEEE
}

\begin{abstract}
It has previously been shown that dispersion compensation can dramatically affect the interchannel crosstalk in a multichannel analog fiber transmission link. In this work, we use a genetic algorithm to find five-segment dispersion maps that yield low crosstalk levels over two octaves of microwave-frequency bandwidth when amplitude modulation is used. The genetic algorithm suggests that optimal dispersion maps have low residual dispersion. Despite the genetic algorithm's ability to optimize dispersion maps with many fibers, it is possible to obtain similar crosstalk levels from a simpler two-segment design whose dispersion is fully compensated.
\end{abstract}

Index Terms-Analog transmission, cross-phase modulation (XPM), crosstalk, intensity modulation, self-phase modulation, wavelength-division multiplexing.

\section{INTRODUCTION}

I $\mathrm{N}$ wavelength-division-multiplexed fiber-optic analog systems, cross-phase modulation (XPM) causes amplitude modulation (AM) in one channel to be transferred to phase modulation (PM) in the others, and chromatic dispersion then converts this PM into undesired AM [1], [2]. Unlike digital systems, where the input optical powers are low and the signal is periodically reamplified, somewhat reducing this effect, analog systems often employ high input optical powers with no reamplification to obtain a high, spurious-free dynamic range [3] over a bandwidth of several octaves. As a result, the effect of interchannel nonlinear crosstalk can be much more severe in these analog systems [2], [4]. Several authors have presented work investigating the impact of interchannel crosstalk, and several have presented novel methods for reducing it by modifying the modulation, detection, or transmission scheme [5]-[7]. However, these methods either require complicated and costly receivers, require parallel fiber links, or work only

Manuscript received June 24, 2007; revised September 11, 2007. Work at the University of Maryland, Baltimore County (UMBC) was supported by the U.S. Naval Research Laboratory. Work at the Naval Research Laboratory (NRL) was supported by the U.S. Office of Naval Research.

B. S. Marks was with the Computer Science and Electrical Engineering Department, University of Maryland, Baltimore County, Baltimore, MD 21250 USA. He is now with the Department of Statistics and the Department of Psychological and Brain Sciences, Indiana University, Bloomington, IN 47405 USA (e-mail: bsmarks@indiana.edu).

C. R. Menyuk is with the Computer Science and Electrical Engineering Department, University of Maryland, Baltimore County, Baltimore, MD 21250 USA.

A. L. Campillo and F. Bucholtz are with the Naval Research Laboratory, Washington, DC 20375 USA.

Color versions of one or more of the figures in this letter are available online at http://ieeexplore.ieee.org.

Digital Object Identifier 10.1109/LPT.2007.913665 over a limited bandwidth. Additionally, simple methods for reducing the crosstalk, such as polarization multiplexing, offer only modest reductions in XPM.

The role of chromatic dispersion in converting PM due to XPM into intensity modulation suggests that tailoring the dispersion map of a link or delay line by splicing together several fibers with different dispersion values may be a useful and simple technique to reduce crosstalk in analog systems. Although some authors have investigated using periodic dispersion compensation to reduce XPM in long or installed links [8]-[10], little work has been presented investigating the possibility of designing a fiber span yielding low crosstalk using an arbitrary dispersion map. In this letter, we use both a genetic algorithm for a five-segment design and a global search for a two-segment design to explore dispersion maps that lead to low interchannel crosstalk over a range of modulation frequencies. We show that while the choice of the fiber segment lengths and dispersions can significantly impact the maximum crosstalk level, the best five-segment system offers no significant advantage over an optimized two-segment system.

\section{METHODS}

Previous work shows that dispersion compensation can be used to reduce crosstalk at individual modulation frequencies [4]. Here, we simulated systems comprising concatenated fiber segments, each with constant dispersion, and we used a genetic algorithm to find configurations that lead to small residual crosstalk over a desired range of modulation frequency. The genetic algorithm attempts to find a global optimum, so that we have some assurance that the crosstalk value corresponding to the configuration found by the algorithm is close to the best achievable value.

We initiate the genetic algorithm simulation by creating a randomly chosen population using the constraints of the problem-a process known as genesis. In our simulation of the crosstalk, each individual is a data structure consisting of the lengths and local chromatic dispersion values of the concatenated fiber segments. We then evaluate the fitness of each individual by computing the negative of the maximum crosstalk in the target range of modulation frequency. We use the negative of the maximum crosstalk so that lower crosstalk corresponds to higher fitness. Once the fitness of each member of the population is evaluated, we rank the members and use a selection process to create the next generation of the population, favoring individuals with high fitness over those with low fitness. The information in the data structure describing each individual is then exchanged among randomly chosen pairs of individuals-crossover - and then a mutation process ensures 
that new randomly chosen parameter values are introduced into the population. After the crossover and mutation steps are complete, the next generation is evaluated for fitness, a new selection process is performed, and the process repeats until the crosstalk level is sufficiently improved.

In the genesis procedure, we choose a population of 100 individuals, whose system parameters are restricted in the following ways. The segments' chromatic dispersion values can take any value in the range of 1 to $50 \mathrm{ps} / \mathrm{nm}-\mathrm{km}$ with either positive or negative sign. The total length of the system is kept fixed at $15 \mathrm{~km}$, an intermediate length for a wideband analog system, while the lengths of each of the fiber segments are allowed to be any fraction of the total length. In our simulations, we fix the fiber loss at $0.2 \mathrm{~dB} / \mathrm{km}$ and the nonlinear coefficient $\gamma$ at $1.55 \mathrm{~W}^{-1} \cdot \mathrm{km}^{-1}$ for all segments in the system. We also use an average launch power of $10 \mathrm{~mW}$ per channel (the launch power required to produce a spur-free dynamic range of approximately $110 \mathrm{~dB}-\mathrm{Hz}^{2 / 3}$ for this link) and the same $200-\mathrm{GHz}$ channel spacing used in our previous work [1], [4].

Next, we evaluate the fitness of each individual by integrating the system of four ordinary differential equations (ODEs) derived in [1] for values of the modulation frequency in the two-octave range of $4-16 \mathrm{GHz}$ with a $1-\mathrm{GHz}$ step size. We use this method because solving the ODE system is significantly computationally faster than solving the full nonlinear Schrödinger partial differential equation. After evaluating the fitness of each individual, the population is sorted by fitness value.

Once the population is sorted, we use a roulette wheel selection process to determine the next generation [11]. In this process, each individual in the population is assigned a wedge on a virtual roulette wheel. The angle subtended by each wedge is proportional to the individual's fitness value minus the minimum fitness of the population, so that the least fit individual is eliminated. A set of 100 independent random numbers uniformly distributed between 0 and $2 \pi$ are then chosen, corresponding to spins of the roulette wheel, and the wedge corresponding to each spin selects the corresponding individual to survive for the next generation. In this process, it is likely to have multiple copies of the most fit individuals.

After selection, we perform crossover, in which randomly selected pairs of individuals exchange parameters of their fiber segments. After pairing, we choose a random number between zero and one and perform crossover on the pair if the number is less than 0.5 . If crossover occurs, we randomly select a fiber segment, referred to as the crossover point, within one of the individuals. The lengths and dispersion values of the fiber segments beyond the crossover point are exchanged between the pair. We have chosen to constrain the system length to remain constant at $15 \mathrm{~km}$. However, after the individuals exchange their parameters, the system length will usually be longer or shorter than $15 \mathrm{~km}$. Therefore, after crossover, we change the lengths of the segments by simply rescaling the system back to $15 \mathrm{~km}$. In performing this rescaling, we change the local dispersion values so that the accumulated dispersion in each fiber segment is maintained. In this way, the crossover process preserves the accumulated dispersion of all fiber segments in the population, although their lengths are changed.
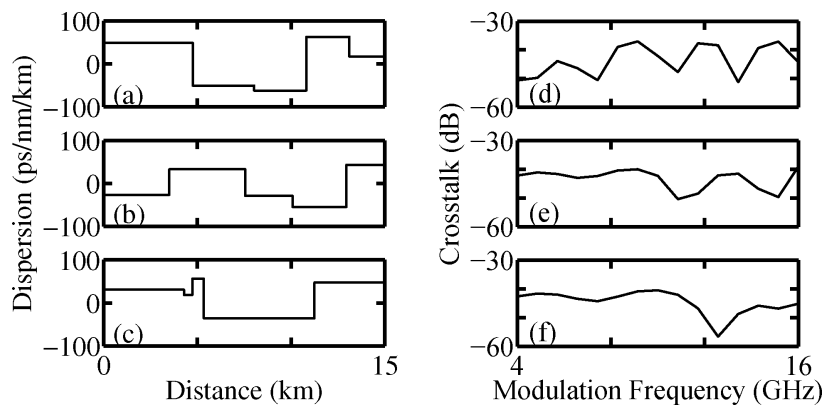

Fig. 1. (a)-(c) Five-fiber dispersion maps resulting from the top three population members after 15 generations of the genetic algorithm, and (d)-(f) the corresponding crosstalk in decibels as a function of modulation frequency. The fitness (negative maximum crosstalk in 4-16 GHz) increases from (a) to (c).

Finally, in the mutation process, we select a random number between zero and one for each fiber segment in the population. If the number is less than 0.15 , we perform a randomization of the local dispersion value for that fiber segment, while keeping its length constant. The new dispersion value is again chosen to be in the range of $1-50 \mathrm{ps} / \mathrm{nm}-\mathrm{km}$ with either a positive or negative sign. While the crossover process is devised to maintain the accumulated dispersion while changing the segments' lengths, the mutation process maintains the segments' lengths while changing the accumulated dispersion.

After several generations, the genetic algorithm eliminates configurations with high maximum crosstalk in favor of those with low crosstalk. Our algorithm finds examples of configurations with very low crosstalk levels over the range of 4-16 GHz.

We computed 15 generations of the population using the genetic algorithm. We verified that the algorithm works by noting that the ensemble average fitness level increases as a function of the generation number. After 15 generations, the average fitness level does not increase appreciably between successive generations. In Fig. 1, we show the dispersion map and the corresponding crosstalk curves for the top three individuals. The dispersion map parameters shown in Fig. 1(a)-(c) look very different from each other.

Despite these differences, the maximum crosstalk achieved in each of the three cases is close to $-40 \mathrm{~dB}$. The first generation of the genetic algorithm yielded a similar result for the lowest maximum crosstalk level. The genetic algorithm was only marginally successful at lowering this best crosstalk level in future generations.

Several salient points arise from these data. First, the dispersion map can indeed be tailored to lower the crosstalk level over a desired range of frequencies. Second, finding an example of a "good" dispersion map is not difficult and does not require a complicated optimization procedure. Finally, while tailoring the dispersion map is beneficial, lowering the crosstalk level further will require changes in the transmitter or receiver design. In sum, it is unlikely that there is a special dispersion map that is dramatically better than all the others. Many dispersion maps produce nearly the same improvement.

Fig. 2 shows a scatter plot of the maximum crosstalk for all 100 individuals in each of the last three generations of the genetic algorithm as a function of the residual dispersion in the line. The most fit individuals appear to be those that have a 


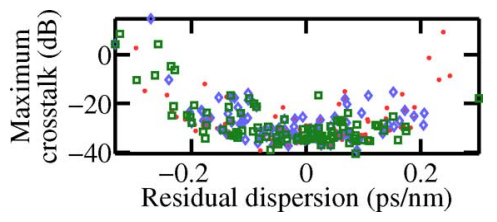

Fig. 2. Scatter plot showing the relationship between the maximum crosstalk and the residual dispersion for all population members in the last three (13-15) generations of the genetic algorithm. Solid circles correspond to generation 13, diamonds correspond to generation 14 , and squares correspond to generation 15 .

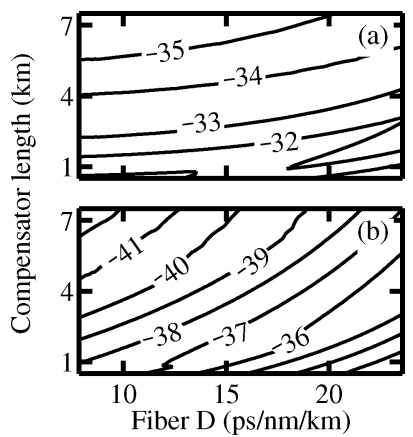

Fig. 3. Contour plots of the maximum crosstalk in the 4- to $16-\mathrm{GHz}$ range as a function of compensator length and fiber dispersion $D$ for a $100 \%$ compensated two-fiber dispersion map. The total length of the dispersion map (fiber + compensator) was $15 \mathrm{~km}$. In (a), the compensator comes before the fiber (pre-compensation), and in (b) the compensator comes after the fiber (post-compensation).

residual dispersion that is close to zero. Therefore, it is natural to restrict our search for optimal dispersion maps to those that have zero net dispersion.

\section{Simulations of Two-Segment DisPersion MAPS}

Motivated by the results of Section II, we next investigated dispersion maps comprised of two segments-a link segment and a compensating segment. As before, the total system length is restricted to $15 \mathrm{~km}$. The compensating segment compensates fully for the dispersion in the link segment so that the residual dispersion is zero. We considered two cases-a pre-compensated system and a post-compensated system. In each case, the length of the compensator is varied between 0.5 and $7.5 \mathrm{~km}$, and the link fiber dispersion is varied between 6 and $27 \mathrm{ps} / \mathrm{nm}-\mathrm{km}$. The attenuation in each segment was set to $0.2 \mathrm{~dB} / \mathrm{km}$.

Fig. 3 shows contour plots of the maximum crosstalk value achieved within the two-octave range of modulation frequencies of 4-16 GHz. In Fig. 3(a), the system is pre-compensated, while in Fig. 3(b), the system is post-compensated. In our simulations, we integrate the ODE system described above for the range of $4-16 \mathrm{GHz}$ with a $1-\mathrm{GHz}$ resolution and interpolate quadratically to find the maximum crosstalk.

Fig. 3 shows that a two-segment dispersion map can achieve a similar crosstalk level as the five-segment dispersion maps that were investigated using the genetic algorithm (about $-42 \mathrm{~dB}$ in these simulations). Additionally, when using either pre- or post-compensation, the configurations that generate the smallest crosstalk correspond to configurations with low dispersion in the link fiber and a compensator length that is the same as the link fiber. Finally, a post-compensated link outperforms a precompensated link. This difference is due to the interaction of the fiber dispersion and nonlinearity in the link. In all cases, the maximum power in the link is at the launch point, and the sign of the dispersion of the first fiber determines the interaction of the dispersion and the nonlinearity. Currently, the reason for the better performance of the post-compensated configuration is not known.

\section{CONCLUSION}

We used a genetic algorithm to find five-segment dispersion maps that minimize the crosstalk over two octaves of bandwidth when AM is used in an analog fiber-optic link. We found that a large number of dispersion maps with widely varying map parameters yielded nearly the same advantage - at best about $-40 \mathrm{~dB}$ for our $15-\mathrm{km}$ system. We also found that it is possible to achieve almost the same advantage using two-segment maps with a zero net dispersion.

Our conclusion is that a significant advantage can be obtained by using appropriate dispersion maps. However, there is little or no advantage to using complex dispersion maps. It does not appear possible to significantly outperform a properly chosen two-segment dispersion map.

\section{REFERENCES}

[1] B. S. Marks, C. R. Menyuk, A. L. Campillo, and F. Bucholtz, "Analysis of interchannel crosstalk in a dispersion-managed analog transmission link," J. Lightw. Technol., vol. 24, no. 6, pp. 2305-2310, Jun. 2006.

[2] M. R. Phillips and D. M. Ott, "Crosstalk due to optical fiber nonlinearities in WDM CATV lightwave systems," J. Lightw. Technol., vol. 17, no. 10, pp. 1782-1792, Oct. 1999.

[3] L. T. Nichols, K. J. Williams, and R. D. Esman, "Optimizing the ultrawide-band photonic link," IEEE Trans. Microw. Theory Tech., vol. 45, no. 8, pp. 1384-1389, Aug. 1997.

[4] A. L. Campillo, E. E. Funk, D. A. Tulchinsky, J. L. Dexter, and K. J. Williams, "Phase performance of an eight-channel wavelength-division-multiplexed analog-delay line," J. Lightw. Technol., vol. 22, no. 2 , pp. 440-447, Feb. 2004

[5] A. L. Campillo, F. Bucholtz, J. L. Dexter, and K. J. Williams, "Crosstalk reduction in wavelength division multiplexed analog links through polarization modulation," in Proc. Conf. Lasers Electro-Optics (CLEO), San Francisco, CA, 2004, Paper CWQ3.

[6] F. S. Yang, M. E. Marhic, and L. G. Kazovsky, "Nonlinear crosstalk and two countermeasures in SCM-WDM optical communication systems," J. Lightw. Technol., vol. 18, no. 4, pp. 512-520, Apr. 2000.

[7] K. K. Y. Wong, M. E. Marhic, and L. G. Kazovsky, "50-dB nonlinear crosstalk suppression in a WDM analog fiber system by complementary modulation and balanced detection," J. Lightw. Technol., vol. 21, no. 2, pp. 500-505, Feb. 2003.

[8] R. Hui, K. R. Demarest, and C. T. Allen, "Cross-phase modulation in multispan WDM optical fiber systems," J. Lightw. Technol., vol. 17, no. 6, pp. 1018-1026, Jun. 1999

[9] J. Chen, H. Kim, and Y. C. Chung, "Cross-phase modulation in shortperiod dispersion managed fiber," IEEE Photon. Technol. Lett., vol. 13, no. 7, pp. 663-665, Jul. 2001.

[10] J. Chen, K. Kim, and Y. C. Chung, "Inter-channel interference in multispan WDM transmissions around perfect dispersion-compensated region,” Opt. Commun., vol. 213, no. 4-6, pp. 367-371, Dec. 1, 2002

[11] Z. Michalewicz, Genetic Algorithms + Data Structures $=$ Evolution Programs. New York: Springer-Verlag, 1998. 